\title{
Implications of the TCGA Genomic Characterization of Papillary Thyroid Carcinoma for Thyroid Pathology: Does Follicular Variant Papillary Thyroid Carcinoma Exist?
}

\author{
Sylvia L. Asa, ${ }^{1}$ Thomas J. Giordano, ${ }^{2}$ and Virginia A. LiVolsi ${ }^{3}$
}

$\mathbf{T}$ HE HISTORY OF THYROID PATHOLOGY has evolved over many years as pathologists identified characteristic morphologic features of tumors that were associated with distinct clinical behaviors. In the 1960s, thyroid tumor classification was relatively simple. Papillary thyroid carcinoma (PTC) was recognized as a tumor with papillary architecture and as a relatively indolent but locally invasive malignancy that had a predominant tendency to spread to regional lymph nodes. Conversely, follicular thyroid carcinoma (FTC) was a follicular-patterned tumor that was usually expansile and encapsulated but invaded through its capsule and into blood vessels to spread hematogenously; carcinomas with mixed papillary and follicular architectures were determined to behave more like PTCs.

Beginning in the 1970s, endocrine pathologists realized that this simplicity did not capture the full complexity of the situation. Following seminal studies of Lindsay (1), the important recognition in 1977 of the value of nuclear features to predict PTC-like behavior in follicular-patterned tumors gave rise to a new entity: the follicular variant of PTC (FVPTC) $(2,3)$.

In 2014, we face a very complex classification of thyroid carcinomas with multiple variants of every major tumor type (4). As in every field, there are pathologists who are "splitters" and subclassify every variant, and there are "lumpers" who prefer to consolidate lesions with similar behaviors and only recognize as important those variants that have clinical significance. While every variant of thyroid cancer has its proponents, there comes a point where differences lose their value by complicating the ability to compare data and thus contribute to appropriate patient care.

Molecular classifications of cancers allow pathologists to step back and recognize the biological basis for similarities and differences in the various subtypes of malignancies. The recent publication of an integrated genomic characterization of papillary thyroid carcinoma by The Cancer Genome Atlas (TCGA) Research Network has done exactly that for the most common endocrine malignancy-PTC (5).

One of the most important outcomes of this study is the validation of morphology as a reflection of tumor biology and an important parameter in determining tumor behavior. The classification of PTCs into $B R A F^{V 600 E}$-like and $R A S$-like tumors with strikingly distinct genomic features has provided validation of the conventional distinction between classical PTCs and FVPTCs. While the former generally fall into the $B R A F^{V 600 E}$-like category, follicular architecture and relatively subtle nuclear atypia are the hallmark of the RAS-like lesions that are classified as FVPTC. The thyroid differentiation scores (TDS) that were determined based on expression of thyroid function genes in this integrated analysis support the clinical evidence that FVPTC displays a gene expression profile that resembles normal thyroid, as we would expect based on its morphology, whereas classical and tall cell PTCs, while still falling within the category of well-differentiated thyroid cancers, are $B R A F^{V 600 E}$-like and show relatively less evidence of thyroid differentiation with lower expression levels of TDS genes.

What the TCGA findings do not address is the distinction between FVPTC and FTC. Although not specifically included in the analysis, FTCs are known to harbor RAS mutations and have frequent copy number changes $(6,7)$. The common detection of the same RAS mutations and arm-level copy number changes in FVPTC raises the question as to whether FTC and FVPTC are truly distinct entities and, importantly, whether the distinction is of biologic and clinical relevance. Moreover, it is almost certain that some of the 99 FVPTCs included in the study would have been diagnosed as FTCs by some experts, since this area is the subject of one of the controversial disputes in pathology $(8,9)$. There is essentially no consensus regarding the nuclear features seen in FVPTC, that is, how florid nuclear atypia must be or how many atypical nuclei in a follicular lesion are required to classify a neoplasm as FVPTC. Nevertheless, the fact remains that thyroid tumors that show complete follicular architecture and grow as expansile and often encapsulated masses harbor $R A S$ or $R A S$-like mutations as the hallmark of their genetic signature.

Pathologists must now reconsider the value of separating these nearly identical lesions into FTC and FVPTC. We propose that the time has come, based on molecular evidence, to do away with the verbose and often confusing terminology

\footnotetext{
${ }^{1}$ Department of Pathology, University Health Network, Toronto, Canada.

${ }^{2}$ Department of Pathology, University of Michigan, Ann Arbor, Michigan.

${ }^{3}$ Department of Pathology, University of Pennsylvania, Philadelphia, Pennsylvania.
} 
"follicular variant of papillary thyroid carcinoma." But what will the implications of this reclassification be?

The implications for PTC will be clear-the terminology will remain applicable to all thyroid carcinomas that have any form of papillary architecture (except for the rare benign papillary lesions known as "papillary hyperplastic nodules" or "follicular adenomas with papillary hyperplastic features", that lack any of the nuclear atypia of PTC and are generally functioning nodules). This classification will be used for all infiltrative lesions, despite follicular architecture that may predominate (10). These $B R A F^{V 600 E}$-like lesions will be expected to behave like classical or tall-cell PTC. However, beyond this separation, the TCGA study illustrates the genetic heterogeneity present in these "true" PTCs that reflects other genetic changes beyond the common driving alterations and/or altered miRNA expression. Much of this genetic heterogeneity is reflected in tumor morphology (e.g., tall cell), and it will require additional validation effortsmorphological and molecular-to establish the best way to uncover these differences in pathology practice. Such work is already underway.

The implications for follicular-patterned lesions and FTC are more onerous. As we recognize that expansile thyroid neoplasms with follicular architecture are all $R A S$-like tumors with high TDS, the distinction between benign, malignant, and aggressive neoplasms remains a challenge. The true predictor of malignant potential in follicular-patterned tumors remains unclear, but may well be one or several of the candidates: nuclear features that were the foundation of FVPTC, the RAS gene mutation signature, capsular invasion, angioinvasion, or some combination of these. While as pathologists we have been rewarded with the validation of papillary-patterned PTC by genomics, we remain humbled by our lack of consensus concerning the nuclear atypia and features of these follicularpatterned tumors. We must also admit our limitations in identifying capsular invasion based on sampling of capsular tissue for examination, since no tumor can undergo complete capsular examination using our routine histologic approaches. Even the criteria for assessing angioinvasion are inconsistent and controversial (11), and as with the capsule of these tumors, there is no way to ensure thorough evaluation of every relevant vessel using histology. Those of us following patients who have developed metastatic carcinoma after the diagnosis of follicular adenoma have studied those tumors carefully. While we often can agree retrospectively that there was nuclear atypia and/or evidence of invasion, there is still difficulty in the pathology community accepting those features prospectively in new cases.

The TCGA study provides us with the opportunity to simplify thyroid cancer classification by putting an end to the complexity of FVPTC. Instead, we should refocus our efforts to move forward with meaningful studies of the complex area of follicular thyroid neoplasia. Ideally, large-scale pangenomic studies like TCGA of benign and malignant follicular tumors will address this problem.

\section{References}

1. Lindsay S 1960 Carcinoma of the Thyroid Gland. A Clinical and Pathological Study of 293 Patients at the University of California Hospital. Charles C Thomas, Springfield, IL.

2. Chem KT, Rosai J 1977 Follicular variant of thyroid papillary carcinoma: a clinicopathologic study of six cases. Am J Surg Pathol 1:123-130.

3. Rosai J, Carcangiu ML, DeLellis RA 1992 Tumors of the Thyroid Gland. Atlas of Tumor Pathology, Third Series, Fascicle 5. Armed Forces Institute of Pathology, Washington, DC.

4. DeLellis RA, Lloyd RV, Heitz PU, Eng C 2004 Pathology and Genetics of Tumours of Endocrine Organs. IARC Press, Lyons, France.

5. Cancer Genome Atlas Network 2014 Integrated genomic characterization of papillary thyroid carcinoma. Cell 159: 676-690.

6. Adeniran AJ, Zhu Z, Gandhi M, Steward DL, Fidler JP, Giordano TJ, Biddinger PW, Nikiforov YE 2006 Correlation between genetic alterations and microscopic features, clinical manifestations, and prognostic characteristics of thyroid papillary carcinomas. Am J Surg Pathol 30:216-222.

7. Wreesmann VB, Ghossein RA, Hezel M, Banerjee D, Shaha AR, Tuttle RM, Shah JP, Rao PH, Singh B 2004 Follicular variant of papillary thyroid carcinoma: genomewide appraisal of a controversial entity. Genes Chromosomes Cancer 40:355-364.

8. Lloyd RV, Erickson LA, Casey MB, Lam KY, Lohse CM, Asa SL, Chan JK, DeLellis RA, Harach HR, Kakudo K, LiVolsi VA, Rosai J, Sebo TJ, Sobrinho-Simoes M, Wenig BM, Lae ME 2004 Observer variation in the diagnosis of follicular variant of papillary thyroid carcinoma. Am J Surg Pathol 28:1336-1340.

9. Elsheikh TM, Asa SL, Chan JK, DeLellis RA, Heffess CS, LiVolsi VA, Wenig BM 2008 Interobserver and intraobserver variation among experts in the diagnosis of thyroid follicular lesions with borderline nuclear features of papillary carcinoma. Am J Clin Pathol 130:736-744.

10. Virk RK, Theoharis CG, Prasad A, Chhieng D, Prasad ML 2014 Morphology predicts BRAF $(\mathrm{V}(6)(0)(0) \mathrm{E})$ mutation in papillary thyroid carcinoma: an interobserver reproducibility study. Virchows Arch 464:435-442.

11. Mete O, Asa SL 2011 Pathological definition and clinical significance of vascular invasion in thyroid carcinomas of follicular epithelial derivation. Mod Pathol 24:1545-1552.

Address correspondence to: Sylvia L. Asa, MD, PhD

Department of Pathology

University Health Network

200 Elizabeth Street

Toronto, Ontario M5G 2 CC4

Canada

E-mail: sylvia.asa@uhn.ca 


\section{This article has been cited by:}

1. Sylvia L. Asa, Ozgur Mete. 2017. Endocrine pathology: past, present and future. Pathology . [Crossref]

2. Giannini Riccardo, Ugolini Clara, Poma Anello Marcello, Urpì Maria, Niccoli Cristina, Elisei Rossella, Chiarugi Massimo, Vitti Paolo, Miccoli Paolo, Basolo Fulvio. 2017. Identification of Two Distinct Molecular Subtypes of Non-Invasive Follicular Neoplasm with Papillary-Like Nuclear Features by Digital RNA Counting. Thyroid 27:10, 1267-1276. [Abstract] [Full Text HTML] [Full Text PDF] [Full Text PDF with Links]

3. Virginia A. LiVolsi, Ezra Baraban, Zubair W. Baloch. 2017. Familial thyroid carcinoma: the road less traveled in thyroid pathology - an update. Diagnostic Histopatbology 23:8, 366-377. [Crossref]

4. Krzysztof Glomski, Vania Nosé, William C. Faquin, Peter M. Sadow. 2017. Metastatic Follicular Thyroid Carcinoma and the Primary Thyroid Gross Examination: Institutional Review of Cases from 1990 to 2015. Endocrine Pathology 28:2, 177-185. [Crossref]

5. Liena Zhao, Dora Dias-Santagata, Peter M. Sadow, William C. Faquin. 2017. Cytological, molecular, and clinical features of noninvasive follicular thyroid neoplasm with papillary-like nuclear features versus invasive forms of follicular variant of papillary thyroid carcinoma. Cancer Cytopathology 125:5, 323-331. [Crossref]

6. Sylvia L. Asa. 2017. The evolution of differentiated thyroid cancer. Pathology 49:3, 229-237. [Crossref]

7. Yong Joon Suh, Hyoun Jong Moon, Ji-Young Choe, Hyo Jin Park. 2017. The Cancer Genome Atlas Validation of Ancillary Tests for Classifying Papillary Thyroid Carcinoma. International Journal of Thyroidology 10:1, 24. [Crossref]

8. Sergei E. Titov, Mikhail K. Ivanov, Elena V. Karpinskaya, Elena V. Tsivlikova, Sergei P. Shevchenko, Yulia A. Veryaskina, Larisa G. Akhmerova, Tatiana L. Poloz, Olesya A. Klimova, Lyudmila F. Gulyaeva, Igor F. Zhimulev, Nikolay N. Kolesnikov. 2016. miRNA profiling, detection of BRAF V600E mutation and RET-PTC1 translocation in patients from Novosibirsk oblast (Russia) with different types of thyroid tumors. BMC Cancer 16:1. . [Crossref]

9. Hasan Gucer, Pelin Bagci, Recep Bedir, Ibrahim Sehitoglu, Ozgur Mete. 2016. The Value of HBME-1 and Claudin-1 Expression Profile in the Distinction of BRAF-Like and RAS-Like Phenotypes in Papillary Thyroid Carcinoma. Endocrine Pathology 27:3, 224-232. [Crossref]

10. Valderrabano Pablo, Montilla-Soler Jaime, Mifsud Mathew, Leon Marino, Centeno Barbara, Khazai Laila, Padhya Tapan, McCaffrey Thomas, Russell Jeffery, McIver Bryan, Otto Kristen. 2016. Hypermetabolism on 18F-Fluorodeoxyglucose Positron Emission Tomography Scan Does Not Influence the Interpretation of Thyroid Cytopathology, and Nodules with a SUVmax $<2.5$ Are Not at Increased Risk for Malignancy. Thyroid 26:9, 1300-1307. [Abstract] [Full Text HTML] [Full Text PDF] [Full Text PDF with Links]

11. Seong-Keun Yoo, Seungbok Lee, Su-jin Kim, Hyeon-Gun Jee, Byoung-Ae Kim, Hyesun Cho, Young Shin Song, Sun Wook Cho, Jae-Kyung Won, Jong-Yeon Shin, Do Joon Park, Jong-Il Kim, Kyu Eun Lee, Young Joo Park, Jeong-Sun Seo. 2016. Comprehensive Analysis of the Transcriptional and Mutational Landscape of Follicular and Papillary Thyroid Cancers. PLOS Genetics 12:8, e1006239. [Crossref]

12. Hershman Jerome M.. 2016. Follicular Variant of Papillary Thyroid Carcinoma (PTC) Has a Better Prognosis Than Classic or Tall-Cell PTC. Clinical Thyroidology 28:3, 77-78. [Citation] [Full Text HTML] [Full Text PDF] [Full Text PDF with Links]

13. Guo-Xia Tong, Qing Chang, Diane Hamele-Bena, John Carew, Richard S. Hoffman, Marina N. Nikiforova, Yuri E. Nikiforov. 2016. Targeted Next-Generation Sequencing Analysis of a Pendred Syndrome-Associated Thyroid Carcinoma. Endocrine Patbology 27:1, 70-75. [Crossref]

14. Jennifer L. Picarsic, Melissa A. Buryk, John Ozolek, Sarangarajan Ranganathan, Sara E. Monaco, Jeffrey P. Simons, Selma F. Witchel, Nursen Gurtunca, Judith Joyce, Shan Zhong, Marina N. Nikiforova, Yuri E. Nikiforov. 2016. Molecular Characterization of Sporadic Pediatric Thyroid Carcinoma with the DNA/RNA ThyroSeq v2 Next-Generation Sequencing Assay. Pediatric and Developmental Pathology 19:2, 115-122. [Crossref]

15. Massimo Santoro, Francesca Carlomagno. Pathogenesis of Thyroid Carcinoma 1-20. [Crossref]

16. Jonathan Lerner, Melanie Goldfarb. 2015. Follicular variant papillary thyroid carcinoma in a pediatric population. Pediatric Blood \& Cancer 62:11, 1942-1946. [Crossref]

17. Thomas J Giordano. 2015. Thyroid genomics: refining diagnosis, prognosis and treatment. International Journal of Endocrine Oncology 2:2, 105-107. [Crossref] 